\title{
Battery-less Hall sensor operated by energy harvesting from a single Wiegand pulse
}

\author{
Yasushi Takemura*, Member, IEEE, Naoya Fujinaga, Akitoshi Takebuchi, and Tsutomu Yamada \\ Division of Electrical and Computer Engineering, Yokohama National University, Yokohama 240-8501, Japan
}

\begin{abstract}
We operated a Hall sensor using energy harvested from a magnetic wire. A battery-less sensor is expected to be a key device for the internet of things (IoT). Magnetization reversal in magnetic wires with bistable magnetization states induces a pulse voltage in a pickup coil. The amplitude of the voltage is independent of the applied field frequency, down to zero. This fast magnetization reversal is accompanied by a large Barkhausen jump, which has been known as the Wiegand effect. Electricity generation using this effect, obtained with twisted FeCoV magnetic wires, was studied. The energy obtained as a single pulse voltage was $600 \mathrm{~nJ}$. The Hall sensor was operated with this pulse voltage. The pulse power of $0.88 \mathrm{~V} / 1.3 \mathrm{~mA}$ was applied to the Hall sensor. The Hall voltage was proportional to the sensing magnetic field of 50-300 $\mathrm{mT}$.
\end{abstract}

Index Terms - Battery-less sensor, energy harvesting, FeCoV wire, Hall sensor, Wiegand effect, Wiegand pulse.

\section{INTRODUCTION}

$\mathrm{M}$ AGNETIZATION reversal in magnetic wires with bistable magnetization states is accompanied by a large Barkhausen jump, which is known as the Wiegand effect [1]. The fast magnetization reversal induces a pulse voltage in a pick-up coil. The amplitude of the voltage is independent of the applied field frequency, down to zero. This phenomenon has been applied in various sensor applications, including rotation sensors and position sensors. Furthermore, Wiegandeffect sensors are normally operated without battery supplies, because they generate a pulse voltage by themselves. Thus, these sensors can be utilized not only as a magnetic sensor generating an output signal, but also as a power generating device. Recently, integrated circuits (ICs) powered by energy harvesting from the Wiegand effect have been made commercially available for a multi-turn counter/encoder [2].

Energy harvesting is attracting much attention for wireless devices, wireless networks, and other applications. These applications are essential for future technology, realizing the internet of things (IoT). In this paper, the battery-less operation of a Hall sensor powered by energy harvesting from the Wiegand effect is discussed. We have reported electricity generation from a vibration-type energy-harvesting element using magnetic wires $[3,4]$. As the pulse voltage induced in a pick-up coil is independent of the applied field frequency, a constant output voltage is generated even by a single event of the extremely slow movement of an excitation magnet. It was found that just a $0.6-\mathrm{mm}$ movement of a $\mathrm{NdFeB}$ magnet was sufficient to generate an output voltage [4]. The operation of the Hall sensor achieved in this study suggests wide potential

Manuscript received April 1, 2015; revised May 15, 2015 and June 1, 2015; accepted July 1, 2015. Date of publication July 10, 2015; date of current version July 31, 2015. (Dates will be inserted by IEEE; "published"

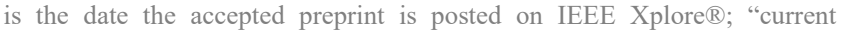
version" is the date the typeset version is posted on Xplore () ).

*Corresponding author: Yasushi Takemura (e-mail: takemura@ynu.ac.jp).

Color versions of one or more of the figures in this paper are available online at http://ieeexplore.iee.org.

Digital Object Identifier (inserted by IEEE). applications of the Wiegand effect for battery-less sensors and devices.

\section{WIEGAND EFFECT AND INDUCED PULSE VOLTAGE FROM FECOV WIRES}

\section{A. Wiegand effect}

The Wiegand effect was initially observed in magnetic wires of NiFe alloys [1]. Vicalloy, with a typical composition of $\mathrm{Fe}_{0.4} \mathrm{Co}_{0.5} \mathrm{~V}_{0.1}$, has been known as the optimum material yielding this effect [5-9], while a large Barkhausen jump in the magnetic bistable FeSiB amorphous wires was also observed $[10,11]$. There have been several publications reporting a Barkhausen jump in magnetic thin films [12-15], but the output voltage is relatively lower [16] than that induced by magnetic wires due to their volume.

\section{B. Magnetic properties of twisted FeCoV wires}

The magnetic wires used in this study were twisted $\mathrm{Fe}_{0.4} \mathrm{Co}_{0.5} \mathrm{~V}_{0.1}$ wires. The wires exhibited different magnetic properties around their center and near surface regions after twisting. The as-prepared wires after twisting and annealing processes were supplied from Nikkoshi Co., Ltd, Japan. Annealing and torsion stress dependences on the magnetic properties of twisted $\mathrm{FeCoV}$ wires were reported in detail by Matsushita et al. [7, 8, 17-19]. Magnetic properties of the $\mathrm{FeCoV}$ wires used in this study are essentially the same as those reported in the articles. The wire reveals a uniaxial magnetic anisotropy along the wire direction. When a torsion stress is applied to the wire, the outer shell near the surface becomes magnetically soft, as shown in Fig. 1. After releasing the stress, the wire exhibits coercive forces of approximately $\mu_{0} H=2 \mathrm{mT}$ in the soft layer and $8 \mathrm{mT}$ in the hard core. Figure 1 shows schematics indicating the magnetic structures of the twisted $\mathrm{FeCoV}$ wire. Details of the magnetic structure are discussed later. 
The magnetization curve (full loop) of the twisted FeCoV wire with a $0.25 \mathrm{~mm}$ diameter and $25 \mathrm{~mm}$ length is shown in Fig. 2(a). The saturation magnetization of the sample was $M_{\mathrm{s}}$ $=1.78 \mathrm{~T}$. The figure also indicates the magnified view of the curve for the applied field, $\mu_{0} H_{\text {ext }}$, from $-6-6 \mathrm{mT}$. Figure $2(\mathrm{~b})$ shows the minor loops of the wire traced with an applied alternating magnetic field of $\mu_{0} H_{\text {ext }}=3,4,5$, and $6 \mathrm{mT}$. These magnetization curves shown in Figs. 2(a) and 2(b) were measured using a vibration sample magnetometer (VSM). A fast magnetization reversal accompanied with a large Barkhausen jump is observed in minor loops in Fig. 2(b), whereas the magnetization reversal is gradual in the full loop indicated by Fig. 2(a). The large Barkhausen jump is due to a magnetization reversal of the magnetically soft layer with a lower coercivity.

\section{Figure 2 HERE}

In order to evaluate the magnetic structure along the radial direction, wires of diameters of $0.16-0.25 \mathrm{~mm}$ were prepared by etching the outer surface using an $\mathrm{FeCl}_{2}$ solution. Figure 3 show a full loop and minor loops for each sample. The magnetization was normalized by that of the wire with a 0.25 $\mathrm{mm}$ diameter. The minor loop was measured by applying an asymmetric field. The magnetic field with a negative direction was varied from $\mu_{0} H_{\mathrm{ext}}=-1,-2$, and $-3 \mathrm{mT}$, whereas the positive field was constant at $\mu_{0} H_{\text {ext }}=15 \mathrm{mT}$. The positive field was enough to almost saturate the hard layer, and the soft and hard layers exhibited parallel alignment. This asymmetric drive field had been used to realize a clear observation of a fast magnetization reversal of the soft layer from the antiparallel to parallel process, against the hard layer $[6,7,8$, 18]. As shown by Fig. 3, the volume of the part of the wire exhibiting the fast magnetization reversal at around $\mu_{0} H_{\mathrm{ext}}=2$ $\mathrm{mT}$ is reduced by decreasing the diameter. This indicates that the etched region near the surface is responsible for the fast magnetization reversal. The magnetization curves corresponding to the hard layer $\left(\mu_{0} H_{\mathrm{ext}}>5 \mathrm{mT}\right)$ were almost constant independent of the diameter. These results suggest that the outer shell layer is magnetically soft with a lower coercivity, and is also responsible for the Barkhausen jump [7, $8,17]$. This suggestion does not agree with the descriptions in some articles that indicate that $\mathrm{FeCoV}$ wires have a soft inner core and a hard outer layer, similar to $\mathrm{NiFe}$ wires $[6,9]$. The volume ratio of the soft layer exhibiting the fast magnetization reversal was calculated to $23 \%$ at a maximum from Fig. 3, which corresponded to the soft later thickness of approximately $0.03 \mathrm{~mm}$ as shown in Fig. 1 .

We had studied the effect of removing the outer layer at both ends by etching, in order to reduce the demagnetizing field and magnetostatic coupling in $\mathrm{FeCoV}$ wires [20]. The fast magnetization of the reversal of the magnetically soft region was strongly affected by the surface of the wire, which also suggested the soft shell layer with a lower coercivity.
Figure 3 HERE

\section{Induced pulse voltage from twisted $\mathrm{FeCoV}$ wires}

Figure 4(a) shows typical waveforms of the output voltage induced in a pickup coil of 3,000 turns wound around the twisted $\mathrm{FeCoV}$ wire $(0.25 \mathrm{~mm}$ in diameter, $25 \mathrm{~mm}$ in length). This was an open-circuit and non-load voltage. The drive field was a symmetric alternating field with $\mu_{0} H_{\text {ext }}=2,3,4,5$, and 6 mT. A fast magnetization reversal was not observed for $\mu_{0} H_{\text {ext }}$ $=2 \mathrm{mT}$. The pulse voltage was induced when the soft layer was reversed with a Barkhausen jump. The full width at the half maximum of the pulse was approximately $20 \mu \mathrm{s}$, which was independent of the drive field intensity. The output voltage was independent of the drive field frequency, down to $1 \mathrm{~Hz}$ [20]. As indicated in Table I, this feature regarding the constant pulse voltage is not achieved by the Hall sensor, magnetoresistance effect (MR) sensor, or electromagnetic induction-type sensor.

\section{TABLE I HERE}

The amplitude of the voltage was increased by increasing the drive field intensity, as indicated by Fig. 4(b). The drive field frequency was lower than $1 \mathrm{~Hz}$ in this measurement. The figure shows both of the positive and negative voltages corresponding to the parallel and antiparallel processes of the magnetization reversal of the soft layer against the hard core. The increase in the voltage is attributed to the increase of the volume of the reversed soft layer. We measured the velocity of the domain wall propagation during the Barkhausen jump. It was approximately $500 \mathrm{~m} / \mathrm{s}$, which was independent of the drive field intensity [21].

\section{Figure 4 HERE}

\section{BATTERY-LESS HALL SENSOR OPERATED BY A WIEGAND PULSE}

\section{A. Energy harvesting from twisted $\mathrm{FeCoV}$ wires}

The use of pulse voltages from twisted FeCoV wires not only for signal generation as a sensing element, but also for electricity generation for operating devices, has been previously considered [22]. We prepared bundled wires to enhance the generated power [23, 24]. The output pulse induced from the bundle of $150 \mathrm{FeCoV}$ wires was $4 \mathrm{~V}$ for 1.2 ms.

Electricity generation using environmental energy or ambient energy, known as energy harvesting, is a promising 
technique for supplying power to wireless devices and wireless networks. Vibration-type energy-generating elements, which convert vibration to electricity, mostly have their own eigen-frequency or resonant frequency $[25,26]$. Vibration at frequency ranges other than the specific frequency generally results in a decrease in the electricity generation efficiency. Electricity generation from a vibration-type energy-harvesting element using an $\mathrm{FeCoV}$ wire was studied [3, 27]. The specific feature of the Wiegand effect can be more advantageous in applications detecting a single event of substantial movement or motion with extremely low speed.

In order to repeat the generation of a pulse voltage, an alternating magnetic field must be applied to the wire. Typically, rotation of a pair of magnets or a single magnet is used as shown in Figs. 5(a) and (b). An alternating magnetic field parallel to the wire direction is applied by the pair of magnets shown in Fig. 5(a). The method of rotating a single magnet shown in Fig. 5(b) is used for a counter/encoder application. It has been also found that a movement of a single magnet is capable of an excitation field of both the positive and negative direction to the wire [27, 28]. This excitation method using a single magnet shown in Fig. 5(c) is useful in vibration-type electricity generation or other applications.

\section{Figure 5 HERE}

\section{B. Electricity generation from twisted $\mathrm{FeCoV}$ wires}

The voltage drop across the load resistor, $R_{\mathrm{L}}$, connected in series with the pickup coil wound around the twisted FeCoV wire was measured as shown in Fig. 6(a). A pick-up coil of 3,000 turns was wound around a twisted FeCoV wire, which was $0.25 \mathrm{~mm}$ in diameter and $20 \mathrm{~mm}$ long. In order to switch the magnetization of the wire, a pair of $\mathrm{NdFeB}$ magnets of $3 \times 3 \times 5 \mathrm{~mm}^{3}$ (a magnetization direction of $5 \mathrm{~mm}$ ) were rotated as shown in Fig. 5(a). By adjusting the distance between the wire and magnet, the magnetic field applied to the wire was set at $\mu_{0} H=6 \mathrm{mT}$. The voltage drop across the load resistor, $R_{\mathrm{L}}$ was measured by varying its resistance. As shown in Fig. 6(a), the voltage drop, $V_{\mathrm{R}}$, which was increased by increasing the resistance, saturated at around $R_{\mathrm{L}}=1 \mathrm{M} \Omega$. This saturated value of $V_{\mathrm{R}} \approx 3.1 \mathrm{~V}$ is equivalent to the Wiegand pulse voltage, which was measured as its open-circuit voltage.

Figure 6(b) shows the energy consumption in the resistor $R_{\mathrm{L}}$ calculated by the time integral of $I_{\mathrm{R}}{ }^{2} R_{\mathrm{L}}$. Maximum energy consumption of approximately $600 \mathrm{~nJ}$ was recorded at $R_{\mathrm{L}}=200$ $\Omega$, which was almost similar to the DC resistance $(171 \Omega)$ of the pickup coil. This slight difference in resistance matching presumably arises from the inductance of the pickup coil.

\section{Operation of Hall sensor by Wiegand pulse}

This Wiegand pulse voltage was applied to a Hall sensor (THS119, Toshiba) through the connected rectifying diodes and resistor $R_{\mathrm{L}}$, as shown in Fig. 7. Considering the possibility of energy harvesting from both the positive and negative pulse voltages, a full-wave rectifier consisting of four Schottky barrier diodes (forward voltage drop, $V_{\mathrm{F}}=0.5 \mathrm{~T}$ at $1 \mathrm{~A}$ ) was used. The internal resistance and other specifications of the Hall sensor are listed in Table II. The resistance of $R_{\mathrm{L}}=680 \Omega$, which was equivalent to the internal resistance (input) of the Hall sensor, was connected in series as an assumed resistance from additional circuit elements.

Figure 7 HERE

\section{TABLE II HERE}

Figure 8 shows the waveforms of the voltage drop across $R_{\mathrm{L}}$, which was essentially the same as that applied to the Hall sensor. During the measurement, a static magnetic field of $B_{\text {in }}=50-300 \mathrm{mT}$ was applied to the Hall sensor, as the sensing magnetic field. It was confirmed that a constant Wiegand pulse voltage of $0.88 \mathrm{~V}$ was applied to the Hall sensor, independent of the sensing field. At half maximum, the full width of the pulses was $29 \mu$ s. This is longer than that measured at the open-circuit voltage shown in Fig. 4(a), which is due to the response of the $L R$ circuit with diodes. Figure 9(a) shows the waveforms of the Hall voltage operated by a single Wiegand pulse. The peak values of these Hall voltages pulses are plotted as a function of the sensing field intensity, in Fig. 9(b). The Hall voltage was proportional to the sensing field applied to the Hall sensor. The figure also shows the Hall voltage measured conventionally with the DC bias current. It was found that the Wiegand pulse voltage supplied a pulse current of $1.3 \mathrm{~mA}$ to the Hall sensor. The sensitivity (161 $\mathrm{mV} / \mathrm{mA} \cdot \mathrm{T}$ ) of the sensing magnetic field agreed well with the specification of the Hall sensor, and can be changed by varying the parameters of the Hall sensor.

The method of energy utilization studied here is categorized as one-shot energy harvesting. A battery-charging using a capacitor is also a practical method for the applications of energy harvesting from the Wiegand effect [29]. 


\section{CONCLUSION}

The operation of a Hall sensor with the electricity generated from the magnetization reversal of a magnetic wire is demonstrated in this paper. The energy obtained from a single Wiegand pulse during the magnetization reversal of the twisted $\mathrm{FeCoV}$ wire was approximately $600 \mathrm{~nJ}$. Understanding the fundamental properties of the magnetization curves, magnetic structure of the wire, and induced pulse voltage in a pickup coil are significant in order to improve the generated energy. As a constant output voltage is generated even by a single event of extremely slow movement of an excitation magnet, this method of energy harvesting is useful not only for vibration-type generators, but also for the future technologies that can be utilized for the IoT. The successful operation of the Hall sensor achieved in this study suggests a wide potential for the application of the Wiegand pulse for battery-less sensors and devices.

\section{ACKNOWLEDGMENT}

The authors would like to express their gratitude to Nikkoshi Co., Ltd, Japan for supplying FeCoV wires used in this study.

\section{REFERENCES}

[1] J. R. Wiegand and M. Velinsky, "Bistable magnetic device," U.S Patent \# 3,820,090, 1974

[2] https://www.ichaus.de/product/iC-PM

[3] A. Takebuchi, N. Kameda, T. Yamada, and Y. Takemura, "Vibrationtype energy harvesting element using a large Barkhausen jump in magnetic wires," 2016 Joint MMM-Intermag Conference, DJ-02, San Diego, Jan, 2016

[4] A. Takebuchi, T. Yamada, and Y. Takemura, "Reduction of vibration amplitude in vibration-type electricity generator using magnetic wire," J. Mag. Soc. Jpn., 41, pp. 34-40, 2017.

[5] P.E. Wigen, "Wiegand wire: new material for magnetic based devices," Electronics, 48, pp. 100-105, 1975.

[6] J.D. Marks, M.J. Sinko, "The Wiegand effect and its automotive applications," Soc. Automot. Eng., 780208, 1978.

[7] A. Matsushita and S. Abe, "Induced pulse voltage in twisted ferromagnetic wire (in Japanese)," IEEJ Trans. A, 99, pp. 46, 1979.

[8] S. Abe and A. Matsushita, "Pulse generating device with twisted ferromagnetic wire," Proc. 1st Sensor Symp., pp. 277-281, 1981.

[9] J.R. Wiegand, "Switchable magnetic device," U.S. Patent \# 4,247,601, 1981.

[10] R. Malmhall, K. Mohri, F. B. Humphrey, T. Manabe, H. Kawamura, J. Yamasaki, and I. Ogasawara, "Bistable magnetization reversal in 50 $\mu \mathrm{m}$ diameter annealed cold-drawn amorphous wires," IEEE Trans. Magn., 23, pp. 3242-3244, 1987.

[11] M. Vázquez, C. Gómez-Polo, D.-X. Chen, and A. Hernando, "Magnetic bistability of amorphous wires and sensor applications," IEEE Trans. Magn., 30, p. 907-912, 1994.

[12] V.I. Malyutin, "New high-speed planar-type thin film pulse magnetic elements," INTERMAG Digest, BQ-14, Seattle, Apr. 1996.

[13] N. Yano, S. Furukawa, K. Oka, I. Ogasawara, J. Yamasaki and F. B. Humphrey, "Large Barkhausen discontinuity of Co-Fe-Si-B amorphous films sputtered on polymer substrate," IEEE Trans. Magn., 32, pp. 4532-4534, 1996.

[14] S. Abe, A. Matsushita, K. Negishi, Y. Baba and M. Naoe, "Generation of large Barkhausen jump in bilayered thin film," IEEE Trans. Magn. 35 , pp. 3634-3636, 1999.

[15] Y. Takemura, T. Inoue, M. Nishimoto and T. Yamada, "Fabrication of zero-speed sensor using weakly coupled $\mathrm{NiFe} / \mathrm{CoFe}$ multilayer films," IEEE Trans. Magn., 40, pp. 2667-2669, 2004.

[16] Y. Takemura and T. Yamada, "Output properties of zero-speed sensors using $\mathrm{FeCoV}$ wire and $\mathrm{NiFe} / \mathrm{CoFe}$ multilayer thin film," IEEE Sens. J., 6, pp. 1186-1190, 2006
[17] S. Abe and A. Matsushita, "Induced pulse voltage in twisted Vicalloy wire with compound magnetic effect," IEEE Trans. Magn., 31, pp. 3152-3154, 1995

[18] S. Abe, A. Matsushita, and M. Naoe, "Dependence of large Barkhausen jump on length of a Vicalloy fine wire with torsion stress," IEEE Trans. Magn., 34, pp. 1318-1320, 1998

[19] S. Abe, A. Matsushita, and M. Naoe, "Annealing and torsion stress effect on magnetic anisotropy and magnetostriction of Vicalloy fine wire," IEEE Trans. Magn., 33, pp. 3916-3918, 1997.

[20] Y. Takemura, T. Aoki, H. Tanaka, T. Yamada, S. Abe, S. Kohno, and H. Nakamura, "Control of demagnetizing field and magnetostatic coupling in $\mathrm{FeCoV}$ wires for zero-speed sensor", IEEE Trans. Magn., 42, pp. 3300-3302, 2006

[21] H. Tanaka, Y. Takemura, S. Abe, S. Kohno, and H. Nakamura, "Constant velocity of domain wall propagation independent of applied field strength in Vicalloy wire," IEEE Trans. Magn., 43, pp. 2397-2399, 2007.

[22] D. J. Dlugos, D. Small, D. A. Siefer, "Wiegand effect energy generator," U.S. Patent \#6,191,687, 2001.

[23] A. Matsushita and Y. Takemura, "Power generating device using compound magnetic wire," J. Appl. Phys., 87, pp. 6307-6309, 2000.

[24] Y. Takemura and A. Matsushita, "Frequency dependence of output voltage generated from bundled compound magnetic wires," IEEE Trans. Magn., 37, pp. 2862-2864, 2001

[25] A. Moure, M.A. Izquierdo Rodríguez, S. Hernández Rueda, A. Gonzalo, F. Rubio-Marcos, D. Urquiza Cuadros, A. Pérez-Lepe, and J.F. Fernández, "Feasible integration in asphalt of piezoelectric cymbals for vibration energy harvesting," Energ. Convers. Manage., 112, pp. 246$253,2016$.

[26] V. Ostasevicius, V. Markevicius, V. Jurenas, M. Zilys, M. Cepenas, L. Kizauskiene, and V. Gyliene, "Cutting tool vibration energy harvesting for wireless sensors applications", Sens. Actu. A-Phys., 233, pp. 310$318,2015$.

[27] R. Serizawa, T. Yamada, S. Masuda, S. Abe, S. Kohno, F. Kaneko, and Y. Takemura, "Energy harvesting derived from magnetization reversal in FeCoV Wire," Proc. IEEE Sens. 2012, pp. 1878-1881, 2012.

[28] T. Kohara, T. Yamada, S. Abe, S. Kohno, F. Kaneko, and Y.Takemura, "Effective excitation by single magnet in rotation sensor and domain wall displacement of FeCoV wire," J. Appl. Phys., 109, 07E531, pp. 1$3,2011$.

[29] S. Saggini, F. Ongaro, L. Corradini, and A. Affanni, "Low-power energy harvesting solutions for wiegand transducers," IEEE J. Emer. Select. Top. Pow. Elect., 3, pp. 766-779, 2015. 
TABLE I

OUTPUT WAVEFORMS OF VARIOUS MAGNETIC SENSORS

\begin{tabular}{lcc}
\hline \hline \multicolumn{1}{c}{ Sensors } & Detection principle & Output waveform \\
High speed & Low speed \\
\hline Hall sensor & Hall effect & Magnetoresistance effect \\
\hline $\begin{array}{l}\text { Electromagnetic } \\
\text { sensor }\end{array}$ & Electromagnetic induction & \\
Wiegand sensor & Large Barkhausen jump &
\end{tabular}

TABLE II

SPECIFICATIONS OF HALL SENSOR USED IN THIS STUDY

\begin{tabular}{lcc}
\hline \multicolumn{1}{c}{ Characteristic } & Symbol & Measured value \\
\hline Internal resistance (Input) & $R_{\text {in }}$ & $680 \Omega$ \\
Internal resistance (Output) & $R_{\text {out }}$ & $950 \Omega$ \\
Hall voltage & $V_{\mathrm{H}}$ & $160 \mathrm{mV} / \mathrm{mA} \cdot \mathrm{T}$ \\
\hline
\end{tabular}

(a) Parallel alignment

(b) Antiparallel alignment

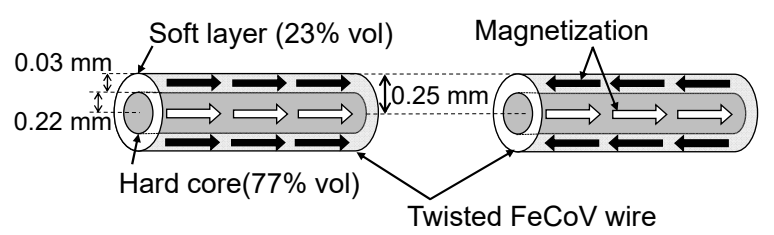

Fig. 1. Schematic of the magnetic structures of the twisted $\mathrm{FeCoV}$ wire with (a) parallel and (b) antiparallel alignments of soft layer and hard core.

(a)

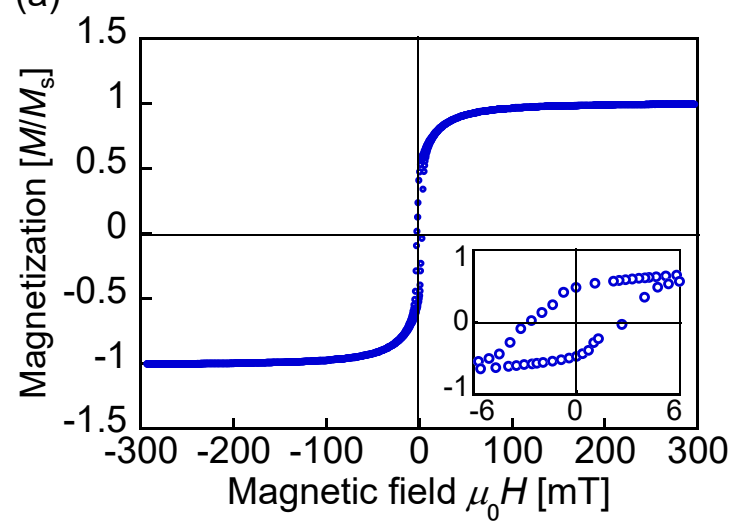

(b)

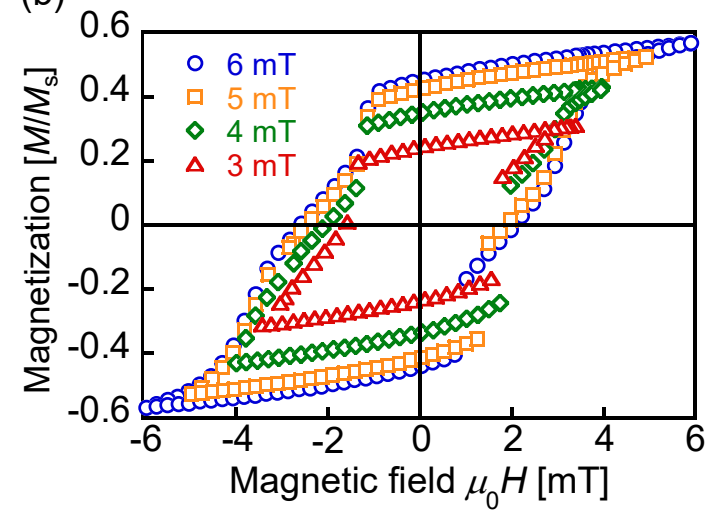

Fig. 2. Magnetization curves of the twisted FeCoV wire $(0.25 \mathrm{~mm}$ in diameter and $25 \mathrm{~mm}$ in length). The applied field intensities were (a) $\mu_{0} H=300 \mathrm{mT}$ (full loop), and (b) $\mu_{0} H=3,4,5$, and $6 \mathrm{mT}$ (minor loops). 


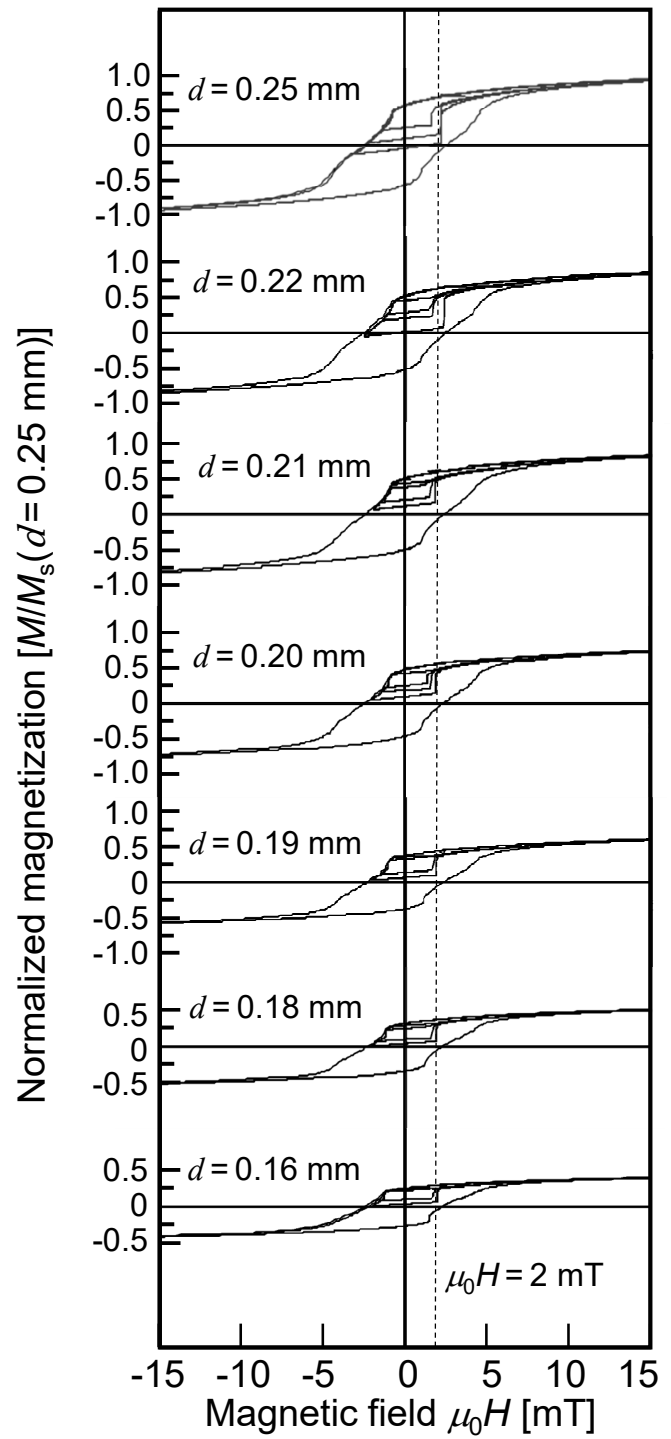

Fig. 3. Magnetization curves of the twisted FeCoV wires, $25 \mathrm{~mm}$ in length. The diameter was varied from $0.25 \mathrm{~mm}$ to $0.16 \mathrm{~mm}$ by etching the outer surface. The magnetization was normalized by that of the wire with a $0.25 \mathrm{~mm}$ diameter. The dotted line indicates the applied magnetic field intensity of $\mu_{0} H=2 \mathrm{mT}$.
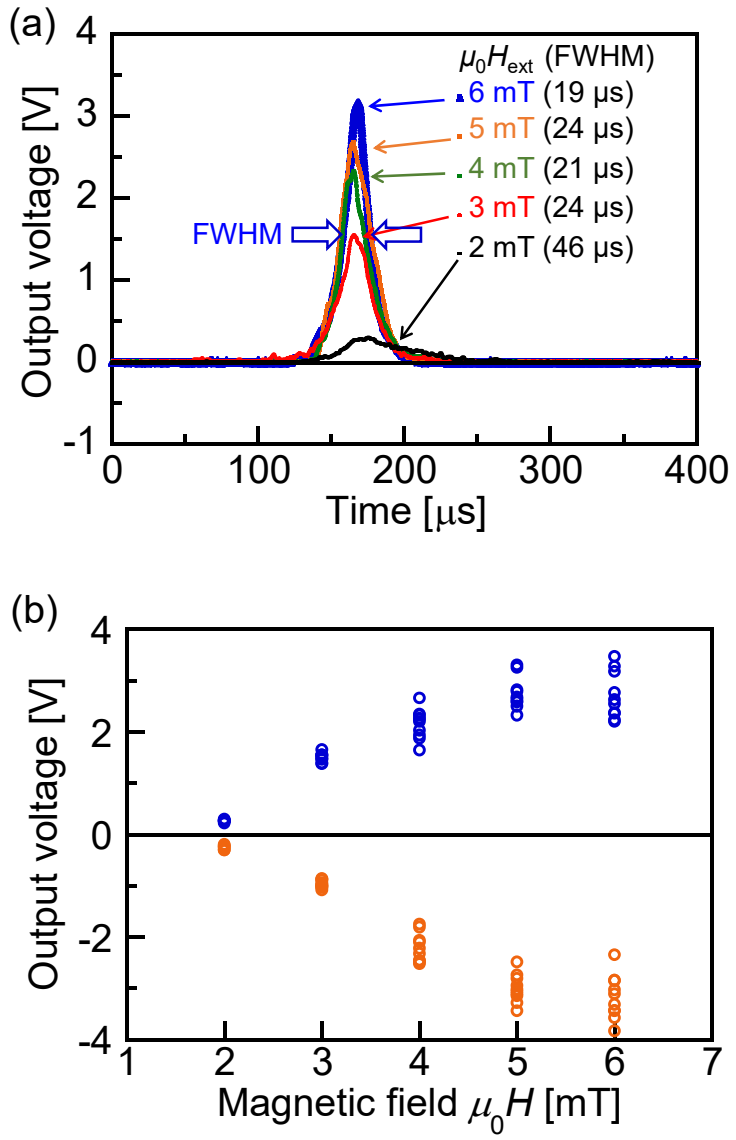

Fig. 4. (a) Waveforms and (b) amplitude of the Wiegand pulse as the output voltage induced in a pickup coil of 3,000 turns wound around the twisted $\mathrm{FeCoV}$ wire. Alternating magnetic fields of $\mu_{0} H=$ $2,3,4,5$, and $6 \mathrm{mT}$ were applied. 
(a)

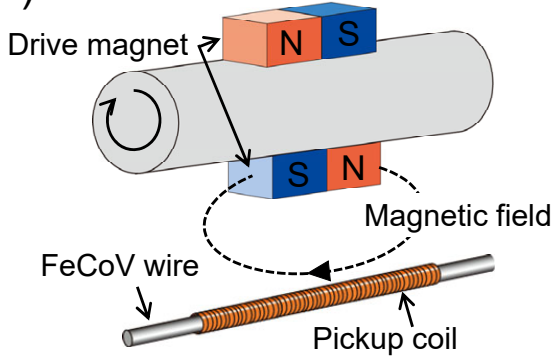

(b)

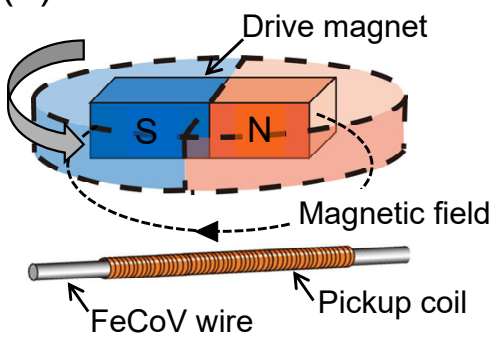

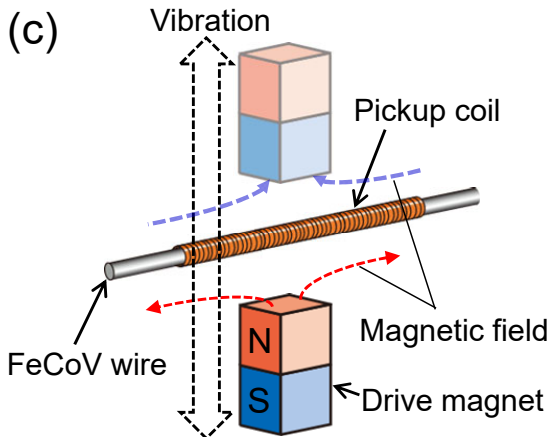

Fig. 5. Variation of the configurations of the FeCoV wire, pickup coil, and drive magnets. (a) Rotation of a pair of magnets, (b) rotation of a single magnet, (c) vibration of a single magnet.

(a)

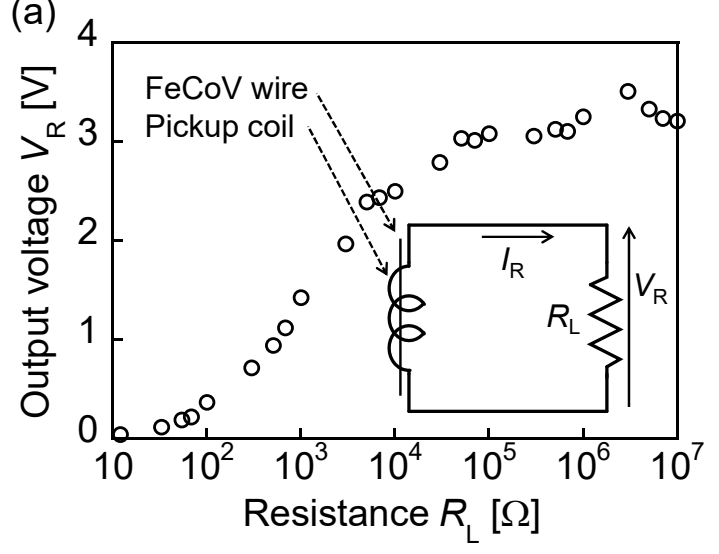

(b)

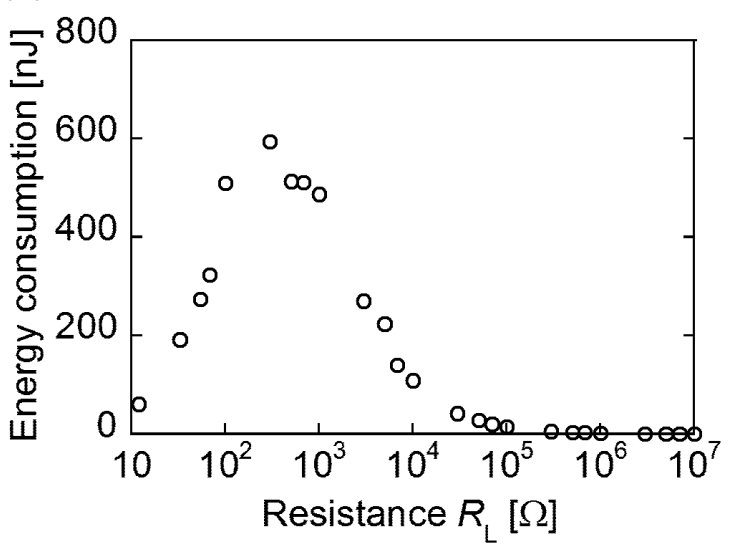

Fig. 6 (a) Voltage drop across the load resistor connected in series with a pickup coil wound around the twisted $\mathrm{FeCoV}$ wire. (b) Energy consumption in the load resistor from a single Wiegand pulse voltage.

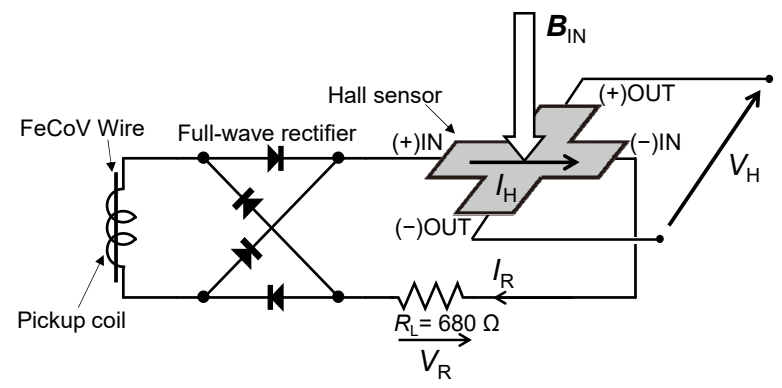

Fig. 7. Circuit for operating a Hall sensor by a single Wiegand pulse voltage. The Hall sensor is connected to the pickup coil wound around the twisted $\mathrm{FeCoV}$ wire through a full-wave rectifier and a load resistor.

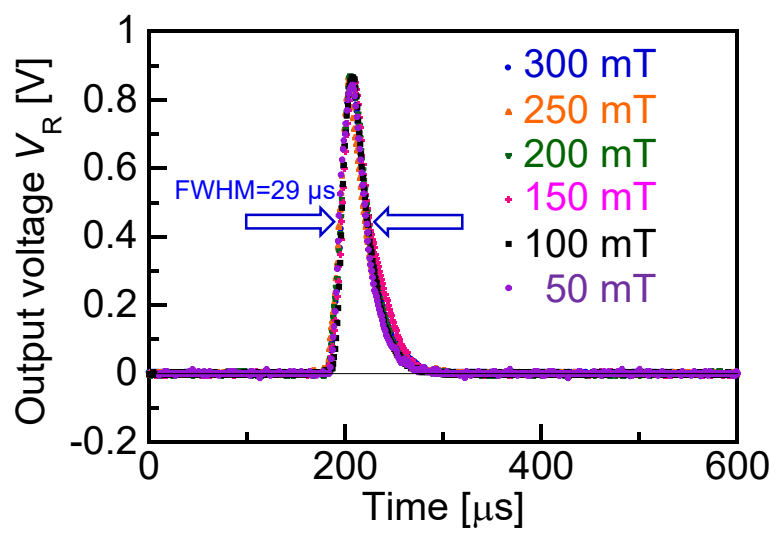

Fig. 8. Waveforms of the voltage drop across the load resistor. This voltage is equivalent to the voltage applied to the Hall sensor. The magnetic flux density applied to the Hall sensor was $B_{\text {in }}=50-300$ $\mathrm{mT}$. 

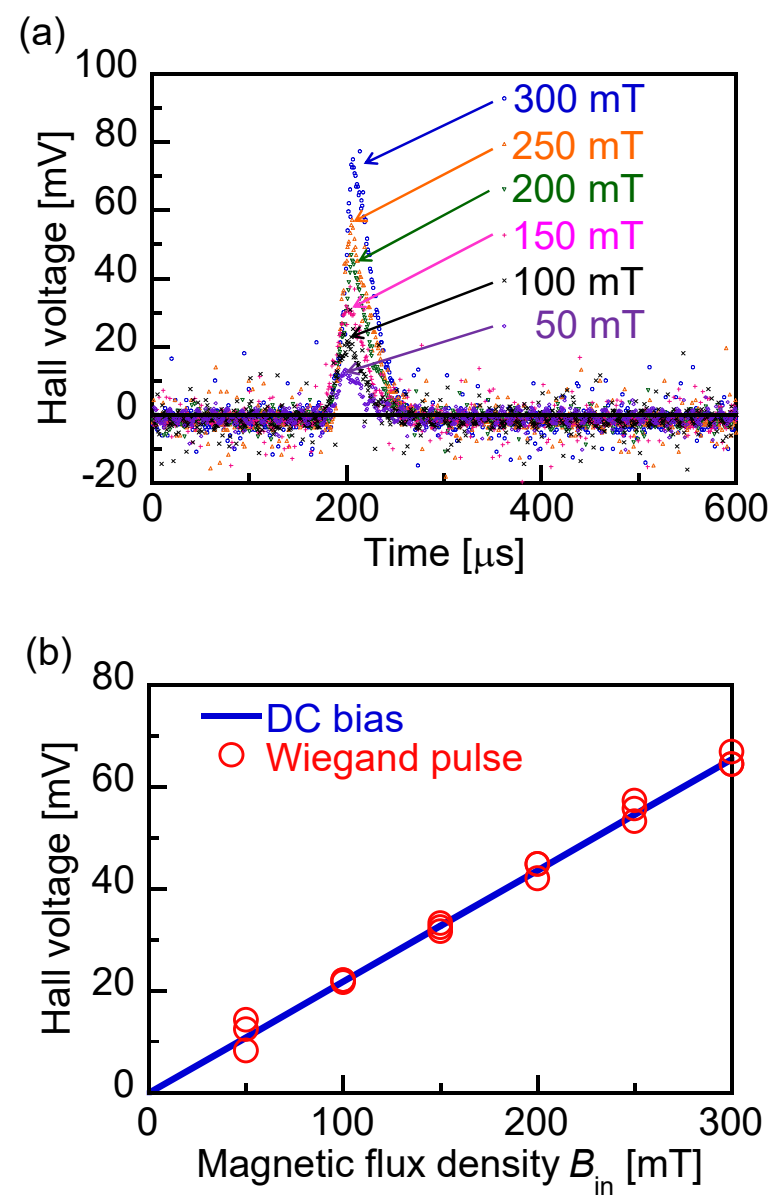

Fig. 9. (a) Waveforms of the Hall voltage operated by a single Wiegand pulse voltage. The magnetic flux density applied to the Hall sensor was $B_{\text {in }}=50-300 \mathrm{mT}$. (b) Hall voltages depending on the applied magnetic flux density. The Hall sensor was operated by a conventional DC bias current of $1.3 \mathrm{~mA}$ or a single Wiegand pulse voltage. 